\title{
Investigation of Physical Properties of Fe(III) Containing Metal-Organic Polymers
}

\author{
A. Yu. Ershova ${ }^{1}$, Minggong Sha ${ }^{2}$, \\ ${ }^{1}$ Moscow Aviation Institute (National Research University), \\ Moscow, Volokolamskoe shosse, 4, 125993, \\ Russia \\ ${ }^{2}$ School of Civil Aviation, Northwestern Polytechnical University (NPU), \\ Xi'an Shaanxi, 127 West Youyi Road, Beilin District, 710072, \\ China
}

Received: March 21, 2021. Revised: August 13, 2021. Accepted: September 10, 2021. Published: September 13, 2021.

\begin{abstract}
In this work, we studied the properties of a specially synthesized organometallic coordination polymer - a porous coordination polymer with biocompatible structural elements based on oxoclusters of iron muconate (III). The samples were investigated by scanning electron microscopy, thermogravimetric analysis combined with differential scanning calorimetry, and the study of lowtemperature nitrogen adsorption of a sample obtained by a modified solvothermal technique.

It is shown that most of the pores of the sample have an average radius of $18,8 \AA \sim 1,88 \mathrm{~nm}$. Also, as a result of the study, it is necessary to conclude that the synthesized material has a developed surface area - it is $512,1 \mathrm{~m}^{2} / \mathrm{g}$ and the pore volume is $\sim 0,48 \mathrm{~cm}^{3} / \mathrm{g}$.

It should be concluded that such materials are promising as components for a new generation of various kinds of functional materials with improved or unique characteristics. It is obvious that further research in this area is important from both fundamental and applied points of view.
\end{abstract}

Keywords-Polymers, nanoparticles, adsorption, mechanical properties.

\section{INTRODUCTION}

$\mathrm{O}$ RGANOMETALLIC coordination polymers are a new developing class of compounds built on the basis of inorganic building blocks consisting of metal ions or their clusters connected to each other through organic linkers in such a way that one-, two- or three-dimensional structures are formed. Organic units, in this case, are mono- or polydentate ligands. Some of these materials can find their application for preparation of magnetically controlled sorbents.

One of the modern achievements of nanotechnology is the creation of nano- and micro-sized composite materials and technologies for their industrial production [1]-[13]. Among these materials, powders of magnetically controlled sorbents play important role in medicine. The name itself defines the most important property of magnetically controlled sorbents the ability to remotely control them using an external magnetic field. The combination of this property with the possibility of drug loading and those biochemical properties that can be endowed with particles with the help of a suitable coating make it possible to create fundamentally new methods of treating various diseases. Therefore, the issues related to the use of magnetically controlled sorbents in general medical practice are relevant. In particular, one of these issues is the development of a methodology for the practical calculation of the parameters of the extracorporeal detoxification system, designing the appropriate composite materials.

Coatings on nano and micro-sized particles can serve for many purposes. First of all, modification of the surface with coatings makes it possible to make the particles compatible with various matrixes [14]-[20]. For medical purposes, the biocompatibility with the environments of a living organism is of crucial importance. It is equally important that coatings can significantly enhance or decrease the sorption properties of magnetically controlled sorbents. This provides prerequisites for the creation of magnetically controlled particles with specific sorption properties. It is also known that the coatings prevent the core from leaching out. The presence of a coating also often facilitates the stabilization of particles in an environment with an alkaline $\mathrm{pH}$ or significant salt concentration. For example, the isoelectric point of $\mathrm{SiO}_{2}$ is reached at $\mathrm{pH} 2-3$. Therefore, the particles coated with silica 
are negatively charged at the $\mathrm{pH}$ of the blood, which causes electrostatic repulsion, which avoids the formation of clumps.

The unique physicochemical properties of magnetically controlled sorbents, noted above and many others, attract great interest of researchers of various specialties around the world. This is evidenced by the growth in the number of scientific publications, patents and innovative works related to both the study of the fundamental properties of magnetically controlled particles and the solution of applied problems associated with the development of methods for their targeted use in medicine and biology.

This work is a continuation of previous works, which presents the results of comparative studies of the structure and sorption capacity of nano- and micro-sized particles to low-, medium- and high-molecular objects [21]-[27].

In addition to being used in devices for the standardized extraction of reagents in biofluid, magnetically controlled sorbents can find no less effective use for introducing useful substances (vitamins, microelements, or other additives) into biofluid. Indeed, the release of the active reagent from the shallow pores of the specified sorbent (a thin layer of the carbon shell) is facilitated, does not require much time and is easily controlled. All this makes the specified process well predictable. Accordingly, the rate of establishment of the equilibrium concentration of the active reagent introduced into the biofluid is high, and the current value of the reagent concentration in the solution is quite accurately determined by the surface density of the reagent and the thermodynamic characteristics of the system, taking into account the Langmuir isotherm, and can be purposefully changed depending on the solution temperature.

Taking into account the works on substantiating the method of practical calculation of the parameters of the extracorporeal detoxification system, it seems appropriate to determine the dependence of the concentration of the adsorbed component in the solution on the characteristics of the type of magnetically controlled sorbents used (mass, sorption capacity). Obtaining quantitative estimates will make it possible to reasonably set the required dosage of magnetically controlled sorbents for a specific detoxification procedure. Important, that temperature conditions can strongly affect the material and its behavior, so, a range of works was devoted to modelling of temperature conditions of materials [28]-[38].

In order to derive the equation of the adsorption isotherm, a number of simplifications are introduced. All the places where the adsorbed particles are fixed are the same, and adsorption on one of them does not affect the state of the other. The interaction between the adsorbed particles is negligible. The adsorption layer is monomolecular, i.e. it consists of one layer of molecules. In this case, the bond of the adsorbate with the adsorbent is sufficiently strong, which excludes the movement of the adsorption complex along the surface of the adsorbent (localized adsorption).

It is rather difficult to predict with accuracy the structure, chemical (reaction ability) and physical (density, conductivity etc.) properties of the future polymer due to the huge variety of organic and inorganic building units [1]-[12]. It should be noted that organic components are capable, depending on the solvent and synthesis conditions, in different ways to bind metal ions and clusters [13]-[29]. But choosing the initial blocks and the method of connecting them, relying on the already known methods of synthesis, it is possible to assume and select the necessary structure of the material and its properties [30]-[42]. The coordination sphere of the metal and the directionality of the bond of donor atoms, as well as the geometry of the bridging ligand, to a large extent affect the structure and properties of the polymer [43]-[80]. Therefore, a particularly important task in the synthesis of MOCP is the control of the interaction of components in the solvent, as well as the correct selection of organic and inorganic components.

\section{STUDY OF THE PROPERTIES OF ORGANOMETALLIC COORDINATION POLYMERS}

The adsorption capacity of any adsorbent is determined primarily by its specific surface area $s_{0}=s / m$, where $\mathrm{s}_{0}$ is the surface area of the adsorbent; $m=V d$ - its mass; $\mathrm{d}$ $\left(\mathrm{kg} / \mathrm{m}^{3}\right)$ - the density of the adsorbent, $\mathrm{V}\left(\mathrm{m}^{3}\right)$ - the volume. From this we obtain $s_{0}=s /(V d)=(1 / d) \delta$, where $\delta=s / V$ is the degree of dispersion (fragmentation) of the adsorbent.

Specific surface area of the adsorbent $\mathrm{s}_{0}$, and, consequently, its adsorption capacity will be the greater, the greater its degree of dispersion $\delta$, or the smaller the linear dimensions of the particles into which the adsorbent is crushed.

Active (well absorbing) adsorbents have a very large specific surface area. For example, the absorbers most often used for scientific purposes and industrial practice - activated carbon, silica gel, zeolites - have $\mathrm{s}_{0}$ up to several hundred and even thousands of square meters per $1 \mathrm{~g}$.

The ability of the adsorbent to absorb the adsorbate is characterized by the amount of adsorption. The amount of adsorption is the excess mass of the adsorbate in the boundary layer over its mass in an equal volume of the environment, referred to the unit surface of the adsorbent.

Sometimes the adsorption value is expressed in moles of adsorbate per $1 \mathrm{~m}^{2}$ (or $1 \mathrm{~cm}^{2}$ ) of the adsorbent surface. Since quite often the surface of the adsorbent is unknown, the value of adsorption is expressed in moles of adsorbate per $1 \mathrm{~g}$ of adsorbent $(\mathrm{mol} / \mathrm{g})$. It is customary to evaluate the process of toxin sorption by the adsorbing surface using the curves of Langmuir sorption isotherms.

As you know, the surface of solids, like liquids, has an excess free Gibbs energy. However, solids, unlike liquids, cannot change the surface area by spontaneous change in shape. Therefore, the tendency to a decrease in the excess surface Gibbs energy in systems where the solid phase (the surface of magnetically controlled sorbents) is in contact with a liquid solution manifests itself mainly in the ability to retain solute molecules on the surface. The latter is possible if the molecules of the solute interact with the surface more strongly 
than with the molecules of the solvent - the liquid to be purified (adsorption).

The dependence of the adsorbed amount of toxin $\Gamma$ on its concentration in solution at a constant temperature is called the adsorption isotherm, a typical form of which is shown below in Fig.1.

The Langmuir adsorption isotherm in coordinates $\Gamma=\Gamma\left(C_{a}\right)$ is a hyperbola and is shown in Fig. 1.

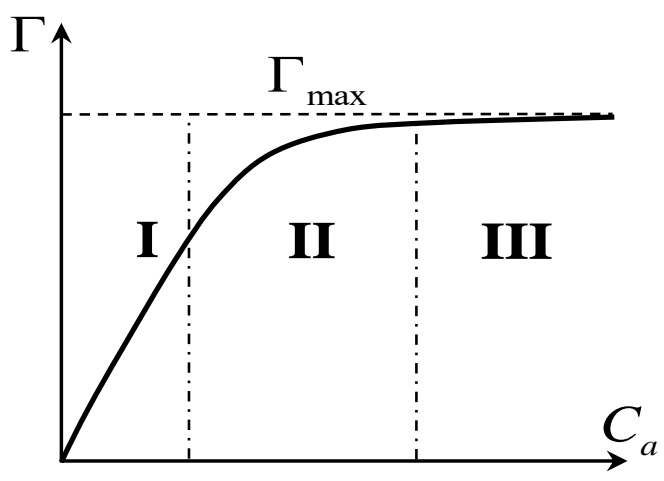

Fig. 1 Adsorption isotherm and its characteristic regions.

During adsorption, the surface of the adsorbent is gradually filled with molecules of the adsorbed substance: first, the most active areas are filled, and then the entire surface. After filling it, molecules can form a second, third, etc. layers. Therefore, a distinction is made between monomolecular and polymolecular adsorption. Given the monomolecularity of the adsorption layer, its thickness is obviously limited. In this case, the amount of toxin absorbed by the sorbent cannot exceed the limiting value $\Gamma=\Gamma_{\max }$.

\section{RESULTS AND DISCUSSION}

One of the main problems in the field of coordination polymers is their thermal stability. Therefore, to study these properties, the resulting compound was subjected to thermal analysis. As can be seen from Figure 2, the thermal decomposition of the sample obtained by the solvothermal method up to $250{ }^{\circ} \mathrm{C}$ occurs monotonically with a slight endoeffect on DSC. Then the DSC curve shows an exo-effect at $288.3{ }^{\circ} \mathrm{C}$. It can be concluded that when the sample is heated to $300{ }^{\circ} \mathrm{C}$, water molecules and solvent residues are removed, accompanied by polymerization. The weight loss corresponds to this when calculated from the found molecular weight of the polymer. Table 1 presents data on the change in the mass of the obtained coordination polymer.

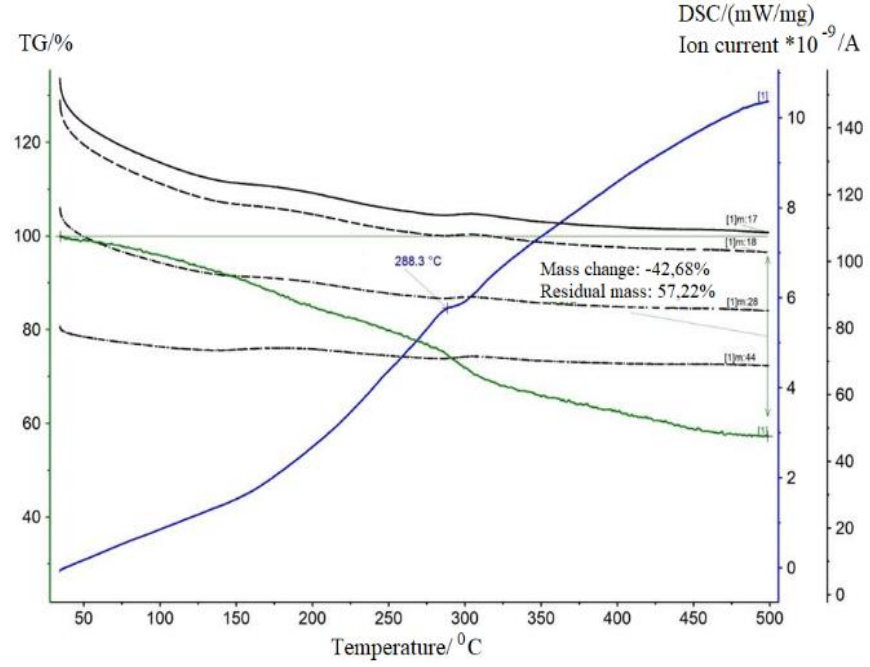

Fig. 2 Thermogravimetric analysis of the obtained coordination polymer combined with differential scanning calorimetry.

Table 1. Results of thermogravimetric analysis of the obtained coordination polymer combined with differential scanning calorimetry.

\begin{tabular}{|c|c|c|c|c|c|c|}
\hline \multirow{2}{*}{ Sample } & \multicolumn{4}{|c|}{ TGA data } & \multirow{2}{*}{$\begin{array}{c}\text { DSC data } \\
\text { Peak }\left[{ }^{\circ} \mathrm{C}\right]\end{array}$} & \multirow{2}{*}{$\begin{array}{c}\text { Residue at } \\
500{ }^{\circ} \mathrm{C} \\
\% \text { wt. }\end{array}$} \\
\hline & $\left.\mathrm{T}_{5 \%}{ }^{[0} \mathrm{C}\right]$ & $\mathrm{T}_{10 \%}\left[{ }^{\circ} \mathrm{C}\right]$ & $\mathrm{T}_{20 \%}\left[{ }^{\circ} \mathrm{C}\right]$ & $\mathrm{T}_{30 \%}\left[{ }^{\circ} \mathrm{C}\right]$ & & \\
\hline $\begin{array}{r}{\left[\mathrm{Fe}_{3} \mathrm{O}\left(\mathrm{C}_{6} \mathrm{H}_{6} \mathrm{O}_{4}\right)_{2}\right.} \\
(\mathrm{H}, \mathrm{O})]\end{array}$ & 110 & 158 & 248 & 309 & $288.3(\max )$ & 57.22 \\
\hline
\end{tabular}

The data from Table 1 allows to calculate the weight of the residual mass of oxoclusters of iron muconate. Scanning electron microscopy was used to confirm the scaffold morphology. According to the results obtained, it can be seen that the topological structure of trinuclear iron acetate (Figure 3 ) is represented by particles of regular shape, characteristic of crystalline substances. Morphology of the iron-containing complex is retained for the resulting coordination polymer (Figure 4).

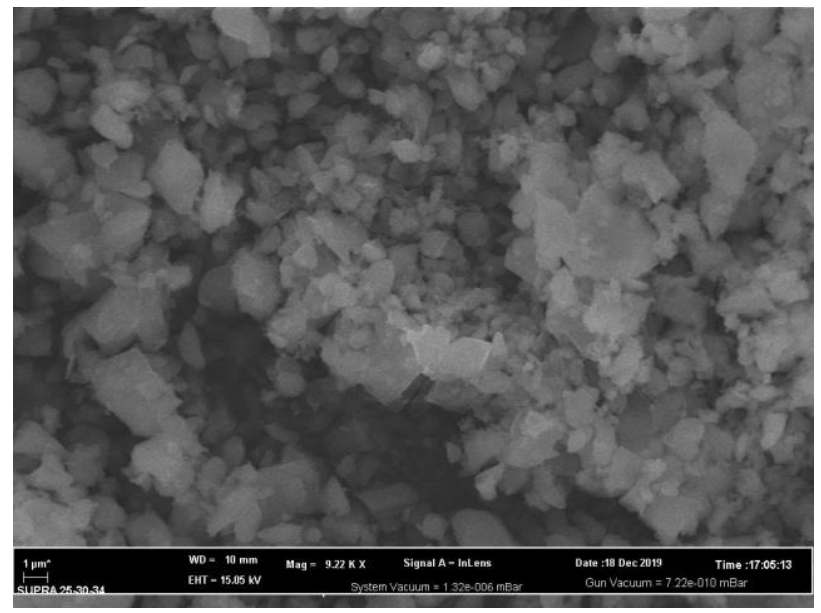

Fig. 3 sem photomicrographs of tricyclic iron acetate. 


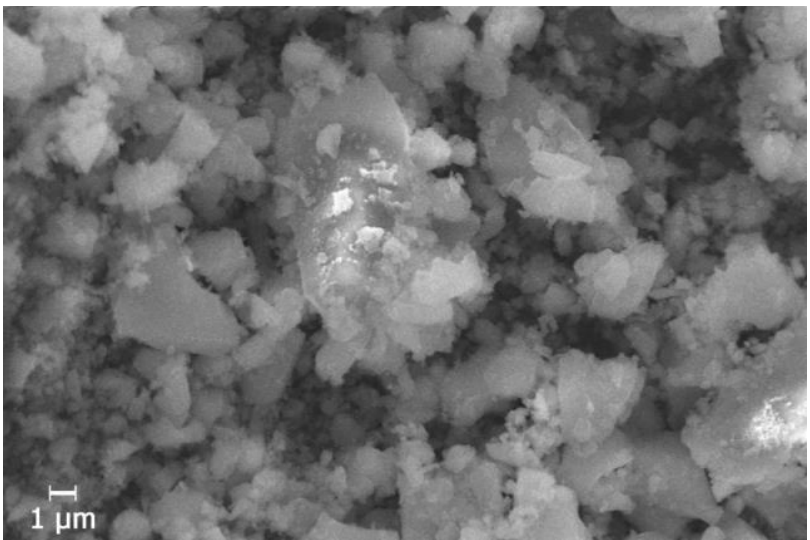

Fig. 4 sem micrographs of the coordination polymer.

Since coordination polymers are characterized by a large surface area, it is important to study the textural characteristics of the synthesized compound. It is necessary in order to characterize the pore size and their distribution, hence, the adsorption capacity of the composite. Figure 5 shows the results of low-temperature nitrogen adsorption of the sample obtained by the modified solvothermal technique. It can be seen that the obtained coordination polymer has an isotherm with a steep rise in the region of low $\mathrm{P} / \mathrm{P}_{0}$ and an almost horizontal section in the saturation region, which indicates polymolecular adsorption on the mesoporous adsorbent.

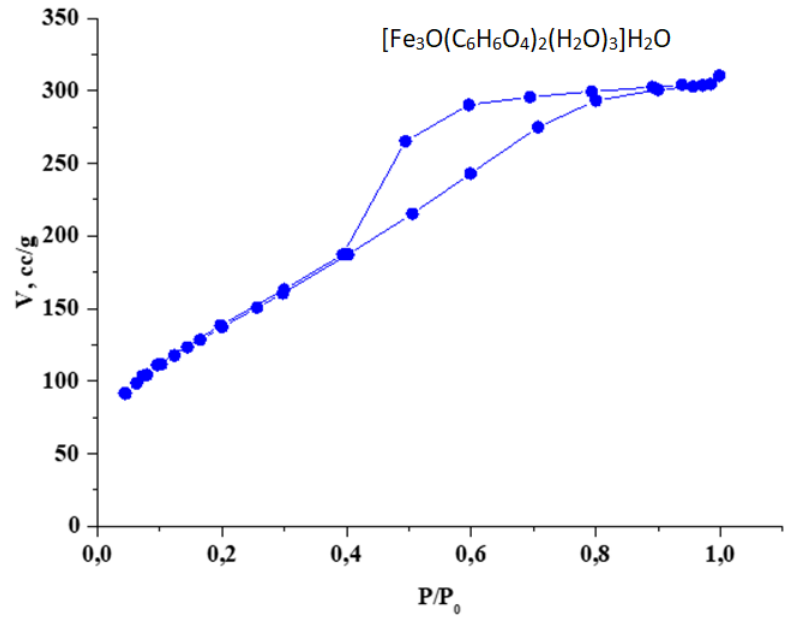

Fig. 5 Adsorption - desorption isotherms of nitrogen for the obtained coordination polymer.

The data obtained as a result of low-temperature $(77 \mathrm{~K})$ nitrogen adsorption confirm that it was possible to reticulate (replace) the acetate linker with the muconic one. Muconic acid, unlike acetate, is capable of binding several inorganic blocks, due to which an extended structure is created. This is confirmed by the surface area obtained as a result of the analysis - it is $512,1 \mathrm{~m}^{2} / \mathrm{g}$ and the pore volume is $\sim 0,48 \mathrm{~cm}^{3} / \mathrm{g}$. As a result, after activation of the sample by thermal vacuum treatment, a mesoporous framework was obtained.

Figure 6 shows the distribution of pore volume along the radii.

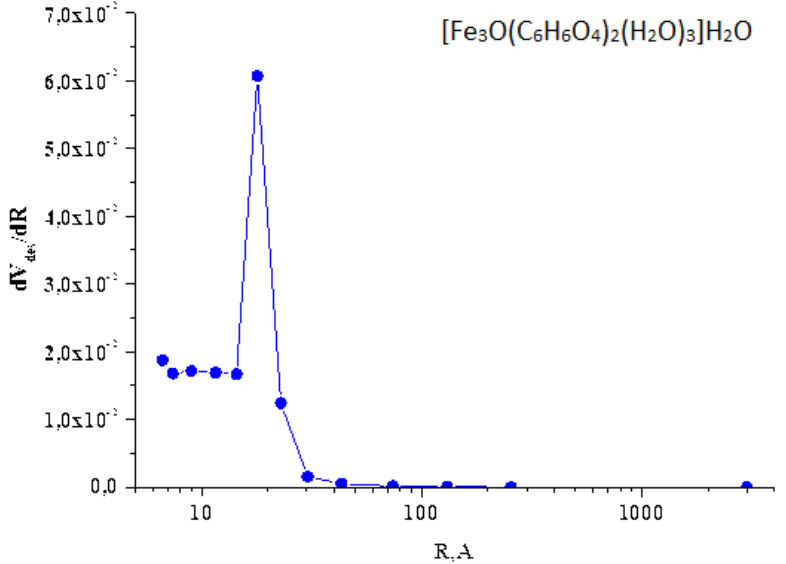

Fig. 6 Differential distribution curve of pore volume along the radii $r$.

\section{CONCLUSION}

From the data obtained, it can be concluded that most of the pores have an average radius of $18,8 \AA \sim 1,88 \mathrm{~nm}$. Also, as a result of the study, it is necessary to conclude that the synthesized material has a developed surface area according to the BET theory (Brunauer, Emmett, Teller) - it is $512,1 \mathrm{~m}^{2} / \mathrm{g}$ and the pore volume is $\sim 0,48 \mathrm{~cm}^{3} / \mathrm{g}$. It should be concluded that such materials are promising as components for a new generation of various kinds of functional materials with improved or unique characteristics. It is obvious that further research in this area is important from both fundamental and practical points of view, since it opens the prospective for tailored fabrication of functional nanocomposites for subsequent application as sorbents and construction materials.

\section{REFERENCES}

[1] L. N. Rabinskiy, S. A. Sitnikov, "Development of technologies for obtaining composite material based on silicone binder for its further use in space electric rocket engines," Periodico Tche Quimica, 15(Special Issue 1), pp. 390-395, 2018.

[2] B. A. Antufev, E. L. Kuznetsova, L. N. Rabinskiy, O. V. Tushavina, "Investigation of a complex stress-strain state of a cylindrical shell with a dynamically collapsing internal elastic base under the influence of temperature fields of various physical nature," Asia Life Sciences, (2), pp. 689-696, 2019.

[3] B. A. Antufev, E. L. Kuznetsova, L. N. Rabinskiy, O. V. Tushavina, "Complex stressed deformed state of a cylindrical shell with a dynamically destructive internal elastic base under the action of temperature fields of various physical nature," Asia Life Sciences, (2), pp. 775782,2019

[4] L. N. Rabinskiy, O. V. Tushavina, "Problems of land reclamation and heat protection of biological objects against contamination by the aviation and rocket launch site," Journal of Environmental Management and Tourism, 10(5), pp. 967-973, 2019.

[5] A. N. Astapov, I. P. Lifanov, L. N. Rabinskiy, "Perspective Heat-Resistant Coating for Protection of 
$\mathrm{Cf} / \mathrm{SiC}$ Composites in Air Plasma Hypersonic Flow," High Temperature, 57(5), pp. 744-752, 2019.

[6] V. N. Dobryanskiy, L. N. Rabinskiy, O. V. Tushavina, "Validation of methodology for modeling effects of loss of stability in thin-walled parts manufactured using SLM technology," Periodico Tche Quimica, 16(33), pp. 650656, 2019.

[7] V. F. Formalev, S. A. Kolesnik, B. A. Garibyan, "Mathematical modeling of heat transfer in anisotropic plate with internal sinks," AIP Conference Proceedings, 2181, 020003, 2019.

[8] O. A. Pashkov, "Influence of Polymer Coatings on the Mechanical Properties of Steel Samples in Tensile and Bending Tests," Turkish Journal of Computer and Mathematics Education (TURCOMAT), vol. 12, no. 5, pp. 542-548, 2021.

[9] O. A. Pashkov, "Investigation of the Effect of Steel Plate Size and Elevated Temperature on Critical Load in Stability Tests," Turkish Journal of Computer and Mathematics Education (TURCOMAT), vol. 12, no. 10, pp. 1657-1663, 2021.

[10] Y. Sun, O. V. Egorova, E. L. Kuznetsova, "Identification of the front angle of a plane acoustic oblique pressure wave on convex surfaces with the use of analytical solution," Journal of the Balkan Tribological Association, 27(2), pp. 189-197, 2021.

[11]N. A. Kucheva, V. Kohlert, "Mathematical modeling methods for estimation the thermophysical properties of heat-protective composite materials," Turkish Journal of Computer and Mathematics Education (TURCOMAT), vol. 12, no. 10, pp. 1606-1612, 2021.

[12] V. F. Formalev, S. A. Kolesnik, B. A. Garibyan, "Heat transfer with absorption in anisotropic thermal protection of high-temperature products," Herald of the Bauman Moscow State Technical University, Series Natural Sciences, (5), p. 35-49, 2019.

[13]O. A. Butusova, "Surface Modification of Titanium Dioxide Microparticles Under Ultrasonic Treatment," International Journal of Pharmaceutical Research, vol. 12, i. 4, pp. 2292-2296, 2020.

[14] O. A. Butusova, "Stabilization of Carbon Microparticles by High-Molecular Surfactants," International Journal of Pharmaceutical Research, vol. 12, Supplementary Issue 2, pp. 1147-1151, 2020.

[15] Yu. V. Ioni, A. Ethiraj, "New Tailor-Made Polymer Stabilizers for Aqueous Dispersions of Hydrophobic Carbon Nanoparticles," International Journal of Pharmaceutical Research, vol. 12, i. 4, pp. 3443-3446, 2020.

[16] Yu. V. Ioni, "Nanoparticles of noble metals on the surface of graphene flakes," Periodico Tche Quimica, vol. 17, no. 36, pp. 1199-1211, 2020.

[17] O. A. Butusova, "Vinyl Ether Copolymers as Stabilizers of Carbon Black Suspensions," International Journal of Pharmaceutical Research, vol. 12, Supplementary Issue 2, pp. 1152-1155, 2020.

[18] M. O. Kaptakov, "Catalytic Desulfuration of Oil Products under Ultrasonic Treatment," International Journal of
Pharmaceutical Research, vol. 12, Supplementary Issue 2, pp. 1838-1843, 2020.

[19] B. A. Garibyan, "Enhancement of Mechanical Properties of Inorganic Glass under Ultrasonic Treatment," International Journal of Pharmaceutical Research, vol. 12, Supplementary Issue 2, pp. 1829-1832, 2020.

[20] M. O. Kaptakov, "Enhancement of Quality of Oil Products under Ultrasonic Treatment," International Journal of Pharmaceutical Research, vol. 12, Supplementary Issue 2, pp. 1851-1855, 2020.

[21]O. A. Butusova, "Adsorption Behaviour of Ethylhydroxyethyl Cellulose on the Surface of Microparticles of Titanium and Ferrous Oxides," International Journal of Pharmaceutical Research, vol. 12, Supplementary Issue 2, pp. 1156-1159, 2020.

[22]A. N. Tarasova, "Vibration-based Method for Mechanochemical Coating Metallic Surfaces," International Journal of Pharmaceutical Research, vol. 12, Supplementary Issue 2, pp. 1160-1168, 2020.

[23]B. A. Garibyan, "Mechanical Properties of Electroconductive Ceramics," International Journal of Pharmaceutical Research, vol. 12, Supplementary Issue 2, pp. 1825-1828, 2020.

[24] M. O. Kaptakov, "Effect of Ultrasonic Treatment on Stability of TiO2 Aqueous Dispersions in Presence of Water-Soluble Polymers," International Journal of Pharmaceutical Research, vol. 12, Supplementary Issue 2, pp. 1821-1824, 2020.

[25] Yu. V. Ioni, "Synthesis of Metal Oxide Nanoparticles and Formation of Nanostructured Layers on Surfaces under Ultrasonic Vibrations," International Journal of Pharmaceutical Research, vol. 12, i. 4, pp. 3432-3435, 2020.

[26] A. N. Tarasova, "Effect of Reagent Concentrations on Equilibria in Water-Soluble Complexes," International Journal of Pharmaceutical Research, vol. 12, Supplementary Issue 2, pp. 1169-1172, 2020.

[27]A. N. Tarasova, "Effect of Vibration on Physical Properties of Polymeric Latexes," International Journal of Pharmaceutical Research, vol. 12, Supplementary Issue 2, pp. 1173-1180, 2020.

[28]N. A. Bulychev, E. L. Kuznetsova, "Ultrasonic Application of Nanostructured Coatings on Metals," Russian Engineering Research, 39 (9), pp. 809-812, 2019.

[29] N. A. Bulychev, V. V. Bodryshev, L. N. Rabinskiy, "Analysis of geometric characteristics of two-phase polymer-solvent systems during the separation of solutions according to the intensity of the image of micrographs," Periodico Tche Quimica, 16(32), pp. 551559, 2019.

[30] N. A. Bulychev, A. V. Ivanov, "Effect of vibration on structure and properties of polymeric membranes," International Journal of Nanotechnology, vol. 16, nos. 6/7/8/9/10, pp. $334-343,2019$.

[31] N. A. Bulychev, A. V. Ivanov, "Nanostructure of OrganicInorganic Composite Materials Based on Polymer Hydrogels," International Journal of Nanotechnology, vol. 16, nos. 6/7/8/9/10, pp. $344-355,2019$. 
[32] N. A. Bulychev, A. V. Ivanov, "Study of Nanostructure of Polymer Adsorption Layers on the Particles Surface of Titanium Dioxide," International Journal of Nanotechnology, vol. 16, nos. 6/7/8/9/10, pp. $356-365$, 2019.

[33] N. A. Bulychev, L. N. Rabinskiy, O. V. Tushavina, "Effect of intense mechanical vibration of ultrasonic frequency on thermal unstable low-temperature plasma," Nanoscience and Technology: An International Journal, 11 (1), pp. 15-21, 2020.

[34]N. A. Bulychev, L. N. Rabinskiy, "Ceramic Nanostructures Obtained by Acoustoplasma Technique," Nanoscience and Technology: An International Journal, 10 (3), pp. 279-286, 2019.

[35] Yu. V. Ioni, A. Ethiraj, "Study of Microparticles Surface Modification by Electrokinetic Potential Measuring," International Journal of Pharmaceutical Research, vol. 12, i. 4, pp. 3436-3439, 2020.

[36] Yu. V. Ioni, "Effect of Ultrasonic Treatment on Properties of Aqueous Dispersions of Inorganic and Organic Particles in Presence of Water-Soluble Polymers," International Journal of Pharmaceutical Research, vol. 12, i. 4, pp. 3440-3442, 2020.

[37] S. A. Kolesnik, N. A. Bulychev, "Numerical analytic method for solving the inverse coefficient problem of heat conduction in anisotropic half-space," Journal of Physics: Conference Series, 1474(1), 012024, 2020.

[38] I. P. Lifanov, A. N. Astapov, V. S. Terentieva. "Deposition of heat-resistant coatings based on the $\mathrm{ZrSi} 2-$ MoSi2-ZrB2 system for protection of non-metallic composite materials in high-speed high-enthalpy gas flows," Journal of Physics: Conference Series, vol. 1713, no. 1, pp. 012025, 2020.

[39]N. A. Kucheva, "Investigation of the mechanical properties of heat-protective highly porous composite materials using the effective medium model," Turkish Journal of Computer and Mathematics Education (TURCOMAT), vol. 12, no. 10, pp. 1613-1621, 2021.

[40] N. A. Kucheva, V. Kohlert, "Analytical solution of the problem of thermoelasticity for a plate heated by a source with a constant heat supply on one surface," Turkish Journal of Computer and Mathematics Education (TURCOMAT), vol. 12, no. 10, pp. 1622-1633, 2021.

[41] I. P. Lifanov, A. A. Yurishcheva, A. N. Astapov, "Hightemperature protective coatings on carbon composites," Russian Engineering Research, vol. 39, no. 9, pp. $804-$ 808, 2019.

[42] A. N. Astapov, I. P. Lifanov, M. V. Prokofiev, "Hightemperature interaction in the $\mathrm{ZrSi} 2-\mathrm{ZrSiO} 4$ system and its mechanism," Russian Metallurgy (Metally), no. 6, pp. $640-646,2019$.

[43] V. F. Formalev, N. A. Bulychev, S. A. Kolesnik, M. A. Kazaryan, "Thermal state of the package of cooled gasdynamic microlasers," Proceedings of SPIE - The International Society for Optical Engineering, 11322, article number 113221B, 2019.

[44] V. F. Formalev, S. A. Kolesnik, B. A. Garibyan, "Analytical solution of the problem of conjugate heat transfer between a gasdynamic boundary layer and anisotropic strip," Herald of the Bauman Moscow State Technical University, Series Natural Sciences, 5(92), pp. 44-59, 2020.

[45]Y. Sun, S. A. Kolesnik, E. L. Kuznetsova, "Mathematical modeling of coupled heat transfer on cooled gas turbine blades," INCAS Bulletin, 12(Special Issue), pp. 193-200, 2020.

[46]I. Kurchatov, N. Bulychev, S. Kolesnik, E. Muravev, "Application of the direct matrix analysis method for calculating the parameters of the luminescence spectra of the iron ion in zinc sulfide crystals," AIP Conference Proceedings, 2181, 020015, 2019.

[47]O. A. Pashkov, "Theoretical calculation of the thickness of interphase zones in the Al-Al2O3 composite," Turkish Journal of Computer and Mathematics Education (TURCOMAT), vol. 12, no. 10, pp. 1672-1677, 2021.

[48] A. V. Perchenok, E. V. Suvorova, A. A. Farmakovskaya, V. Kohlert, "Application of vinyl ether copolymers for surface modification of carbon black," International Journal of Circuits, Systems and Signal Processing, vol. 15, pp. 1300-1304, 2021.

[49] A. V. Perchenok, E. V. Suvorova, A. A. Farmakovskaya, V. Kohlert, "Stabilization of aqueous dispersions of inorganic microparticles under mechanical activation," WSEAS Transactions on Applied and Theoretical Mechanics, vol. 16, pp. 127-133, 2021.

[50]O. A. Pashkov, "Experimental and Theoretical Study of Mechanical Properties of Matrix Composite Materials," Turkish Journal of Computer and Mathematics Education (TURCOMAT), vol. 12, no. 10, pp. 1678-1684, 2021.

[51] P. F. Pronina, O.V. Tushavina, E. I. Starovoitov, "Study of the radiation situation in moscow by investigating elastoplastic bodies in a neutron flux taking into account thermal effects," Periodico Tche Quimica, 17(35), pp. 753-764, 2020.

[52]A. A. Orekhov, Y. A. Utkin, P. F. Pronina, "Determination of deformation in mesh composite structure under the action of compressive loads," Periodico Tche Quimica, 17(35), pp. 599-608, 2020.

[53] A. V. Babaytsev, L. N. Rabinskiy, K. T. Aung, "Investigation of the contact zone of a cylindrical shell located between two parallel rigid plates with a gap," INCAS Bulletin, 12(Special Issue), pp. 43-52, 2020.

[54] V. G. Dmitriev, O. V. Egorova, E. I. Starovoitov, "Particularities of mathematical modeling of deformation processes for arched and panel designs of composites with large displacements and rotation angles," INCAS Bulletin, 12(Special Issue), pp. 53-66, 2020.

[55]O. V. Egorova, E. I. Starovoitov, "Non-stationary diffraction problem of a plane oblique pressure wave on the shell in the form of a hyperbolic cylinder taking into account the dissipation effect," INCAS Bulletin, 12(Special Issue), pp. 67-77, 2020.

[56] O. V. Tushavina, "Coupled heat transfer between a viscous shock gasdynamic layer and a transversely streamlined anisotropic half-space," INCAS Bulletin, 12 (Special Issue), pp. 211-220, 2020. 
[57] S. Vakhneev, E. Starovoitov, "Damping of circular composite viscoelastic plate vibration under neutron irradiation," Journal of Applied Engineering Science, 18(4), pp. 699-704, 2020.

[58] O. A. Butusova, "Design and Properties of Magnetically Controlled Sorbents," Turkish Journal of Computer and Mathematics Education (TURCOMAT), vol. 12, no. 5, pp. 515-519, 2021.

[59] G. A. Kalugina, A. V. Ryapukhin, "Impact of the 2020 Pandemic on Russian Aviation," Russian Engineering Research, vol. 41. no. 7, pp. 627-630, 2021.

[60]R. N. Zaripov, I. M. Murakaev, A. V. Ryapukhin, "Development of the Organization's Key Performance Indicators System in Order to Improve the Effectiveness of Its Human Capital and Risk Management," TEM Journal, vol. 10, no. 1, pp. 298-302, 2021.

[61]A. A. Kalugin, G. A. Kalugina, A. V. Ryapukhin, "Informational Support for the Sale of Passenger Aircraft," Russian Engineering Research, vol. 41, no. 2, pp. 183-187, 2021.

[62] R. N. Zaripov, I. M. Murakaev, S. V. Novikov, A. V. Ryapukhin, "Corporate Structure for Innovative Enterprises," Russian Engineering Research, vol. 40, no. 2, pp. 137-139, 2020.

[63]A. Y. Burova, "Concept of multistage discrete fourier transform without performing multiplications" Journal of Physics: Conference Series, vol. 1889, no. 2, 022003, 2021.

[64]A. Burova, "Reducing the Error of Digital Algorithms for Deductive Signal Processing Based on Their Multi-Stage Discrete Fourier Transform by the Difference Digital Filters," 22th International Conference on Digital Signal Processing and its Applications, DSPA 2020, no. $9213275,2020$.

[65]A. Y. Burova, "Minimization of asymmetry of thrust of dual-flow turbojet engines of airliner in accordance with the results of system analysis of thrust parameters," Asia Life Sciences, no. 2, pp. 629-643, 2019.

[66] O. A. Butusova, "Application of Magnetically Controlled Sorbents for Detoxication," Turkish Journal of Computer and Mathematics Education (TURCOMAT), vol. 12, no. 5, pp. 520-524, 2021.

[67] V. A. Pogodin, L. N. Rabinskii, S. A. Sitnikov, "3D Printing of Components for the Gas-Discharge Chamber of Electric Rocket Engines," Russian Engineering Research, vol. 39, no. 9, pp. 797-799, 2019.

[68] Y. K. Kyaw, E. L. Kuznetsova, A. V. Makarenko "Complex mathematical modelling of mechatronic modules of promising mobile objects," INCAS Bulletin, 12(Special Issue), pp. 91-98, 2020.

[69] L. E. Kuznetsova, V. G. Fedotenkov, "Dynamics of a spherical enclosure in a liquid during ultrasonic cavitation," Journal of Applied Engineering Science, 18(4), pp. $681-686,2020$.
[70]A. V. Makarenko, E. L. Kuznetsova, "Energy-Efficient Actuator for the Control System of Promising Vehicles," Russian Engineering Research, 39(9), pp. 776-779, 2019.

[71]M. O. Kaptakov, "Effect of Thin Polymer Layers on Mechanical Properties of Metal Surfaces," Turkish Journal of Computer and Mathematics Education (TURCOMAT), vol. 12, no. 5, pp. 525-529, 2021.

[72]B. A. Garibyan, "Determination of the Elastic Modulus of the Coating Using a Spherical Indenter," Turkish Journal of Computer and Mathematics Education (TURCOMAT), vol. 12, no. 10, pp. 1594-1600, 2021.

[73] M. O. Kaptakov, "Modelling of Mechanical Properties of Metal Plates with Polymer Coatings," Turkish Journal of Computer and Mathematics Education (TURCOMAT), vol. 12, no. 5, pp. 530-534, 2021.

[74] B. A. Garibyan, "Theoretical Estimations of Influence of Polymer Coatings on the Elastic Modulus and Ultimate Strength of Steel Samples," Turkish Journal of Computer and Mathematics Education (TURCOMAT), vol. 12, no. 10, pp. 1651-1656, 2021.

[75] M. O. Kaptakov, "Investigation of Effective Mechanical Characteristics of Nanomodified Carbon-Epoxide Composite by Numerical and Analytical Methods," Turkish Journal of Computer and Mathematics Education (TURCOMAT), vol. 12, no. 5, pp. 535-541, 2021.

[76] M. O. Kaptakov, "Obtaining of Carbon Fibers Based Composite Materials and Study of Their Mechanical Properties," Turkish Journal of Computer and Mathematics Education (TURCOMAT), vol. 12, no. 10, pp. 1601-1605, 2021.

[77]E. L. Kuznetsova, A. V. Makarenko, "Mathematic simulation of energy-efficient power supply sources for mechatronic modules of promising mobile objects," Periodico Tche Quimica, 15(Special Issue 1), pp. 330338, 2018.

[78] Y. Li, A. M. Arutiunian, E. L. Kuznetsova, G. V. Fedotenkov, "Method for solving plane unsteady contact problems for rigid stamp and elastic half-space with a cavity of arbitrary geometry and location," INCAS Bulletin, 12(Special Issue), pp. 99-113, 2020.

[79] E. L. Kuznetsova, G. V. Fedotenkov, E. I. Starovoitov, "Methods of diagnostic of pipe mechanical damage using functional analysis, neural networks and method of finite elements," INCAS Bulletin, 2020, 12(Special Issue), p. 79-90.

[80]Y. K. Kyaw, P. F. Pronina, P. O. Polyakov, "Mathematical modelling of the effect of heat fluxes from external sources on the surface of spacecraft," Journal of Applied Engineering Science, 18(4), pp. 732-736, 2020.

\section{Creative Commons Attribution License 4.0 (Attribution 4.0 International, CC BY 4.0)}

This article is published under the terms of the Creative Commons Attribution License 4.0 https://creativecommons.org/licenses/by/4.0/deed.en US 